\title{
Respiratory muscle dysfunction in animal models of hypoxic disease: antioxidant therapy goes from strength to strength
}

This article was published in the following Dove Press journal:

Hypoxia

14 July 2017

Number of times this article has been viewed

\author{
Ken D O'Halloran' \\ Philip Lewis ${ }^{2}$ \\ 'Department of Physiology, School \\ of Medicine, University College \\ Cork, Cork, Ireland; ${ }^{2}$ Institute and \\ Policlinic for Occupational Medicine, \\ Environmental Medicine and \\ Preventative Research, University \\ Hospital of Cologne, Germany
}

\begin{abstract}
The striated muscles of breathing play a critical role in respiratory homeostasis governing blood oxygenation and $\mathrm{pH}$ regulation. Upper airway dilator and thoracic pump muscles retain a remarkable capacity for plasticity throughout life, both in health and disease states. Hypoxia, whatever the cause, is a potent driver of respiratory muscle remodeling with evidence of adaptive and maladaptive outcomes for system performance. The pattern, duration, and intensity of hypoxia are key determinants of respiratory muscle structural-, metabolic-, and functional responses and adaptation. Age and sex also influence respiratory muscle tolerance of hypoxia. Redox stress emerges as the principal protagonist driving respiratory muscle malady in rodent models of hypoxic disease. There is a growing body of evidence demonstrating that antioxidant intervention alleviates hypoxiainduced respiratory muscle dysfunction, and that $\mathrm{N}$-acetyl cysteine, approved for use in humans, is highly effective in preventing hypoxia-induced respiratory muscle weakness and fatigue. We posit that oxygen homeostasis is a key driver of respiratory muscle form and function. Hypoxic stress is likely a major contributor to respiratory muscle malaise in diseases of the lungs and respiratory control network. Animal studies provide an evidence base in strong support of the need to explore adjunctive antioxidant therapies for muscle dysfunction in human respiratory disease.
\end{abstract}

Keywords: respiratory muscle, diaphragm, upper airway, hypoxia, antioxidants, N-acetylcysteine, OSA, COPD

\section{Introduction}

This review article focuses on the experimental evidence supporting a fundamentally important role for oxygen homeostasis in shaping respiratory muscle form and function. We consider studies employing rodent models of hypoxia relevant to human respiratory disease. Chronic intermittent hypoxia $(\mathrm{CIH})$ is a dominant feature of human sleep-disordered breathing. Chronic sustained hypoxia (CSH) has relevance to environmental stress at high altitude and chronic lung diseases. We review studies exploring the mechanisms underpinning aberrant respiratory muscle remodeling following hypoxic exposure with a focus on studies employing antioxidant strategies to combat hypoxia-induced respiratory muscle dysfunction. We compare and discuss different respiratory muscles and hypoxic paradigms in various animal models. The emerging evidence strongly points to the need to explore the efficacy of adjunctive antioxidant therapies for respiratory muscle dysfunction in human respiratory disease.

\section{Respiratory muscles: function, form, and plasticity}

The respiratory muscles are the final effector organs of a complex neuromuscular control network that governs respiratory homeostasis ensuring adequate arterial blood
( Department of Physiology, School of Medicine, University College Cork, Road, Cork, Ireland

Tel +3532l 4205433

Email k.ohalloran@ucc.ie 
gas and $\mathrm{pH}$ regulation. Neuromechanical coupling underpins ventilatory and nonventilatory functions such as protective airway clearance maneuvers and highly complex behaviors such as phonation. Activation of chest pump muscles, principally the diaphragm and intercostal muscles, generates dynamic cyclical changes in thoracic volume and pressure differentials sufficient to encourage airflow. ${ }^{1-4}$ This allows for effective gas exchange between the external environment and the alveoli, the highly vascular functional units of gas exchange in the lungs. Beyond the principal and accessory muscles of breathing that facilitate pulmonary ventilation across a range of activities are several other complementary muscles of the upper airways. These muscles serve a critical function in determining airway tone and caliber. ${ }^{5,6}$ These dilator and constrictor muscles regulate upper airways resistance, thus gating inspiratory and expiratory airflow, defending airway patency on a breath-by-breath basis.

There is relatively tight structure-function coupling in respiratory muscles. The diaphragm is a thin, dome-shaped muscle with a mixed muscle fiber-type complement befitting the physiological roles of the muscle. ${ }^{7,8}$ Basal breathing requires rhythmic activation of the diaphragm at a low level of maximum force-generating capacity. ${ }^{3,4}$ The high oxidative capacity of the diaphragm muscle fibers ${ }^{7,8}$ confers considerable fatigue resistance, a useful trait for the most active striated muscle in the body. Fast glycolytic fibers are also present ${ }^{7,8}$ and are recruited for near-maximal activation during airway obstruction, sneezing, and exacerbations of chronic respiratory disease. ${ }^{9,10}$ In contrast, upper airway dilator muscles are characterized by a predominant expression of fast glycolytic fibers, ${ }^{11,12}$ which may be important in preventing/overcoming pharyngeal collapse and maintaining airway patency in addition to nonrespiratory functions in deglutition and speech. ${ }^{5,6}$ While the fast phenotype of upper airway muscles facilitates powerful contractions, it renders them more vulnerable to fatigue. Notwithstanding the essential function of respiratory muscles to sustain life, the pump and airway dilator striated muscles of breathing retain considerable capacity for structural and functional plasticity in response to changes in functional demands. This extends far beyond developmental periods wherein temporal fibertype and functional changes are well recognized. ${ }^{13-17}$ Indeed, diaphragm and upper airway dilator muscles show remarkable capacity for plasticity, as impressive as that documented in limb skeletal muscles in response to a variety of stressors in health and disease. ${ }^{18}$

Highly malleable respiratory muscles might confer advantage in allowing organisms to respond to physiological challenges (e.g., exercise, pregnancy, development, and aging); however, it is increasingly apparent that aberrant remodeling has the capacity to limit ventilatory performance and contribute substantively to patient morbidity and mortality. Diaphragm dysfunction is described in critically ill patients and related animal models, ${ }^{19-22}$ and in chronic respiratory conditions such as chronic obstructive pulmonary disease (COPD). ${ }^{23,24}$ Upper airway muscle dysfunction is implicated in the pathophysiology of obstructive sleep apnea syndrome (OSAS). ${ }^{25-27}$ Importantly, different paradigms of hypoxia feature in such respiratory diseases.

\section{Hypoxia is a consequence and cause of respiratory morbidity}

Hypoxia arises in most tissues when there is inadequate oxygenation to meet functional demands. In healthy individuals, hypoxia can manifest at higher altitudes (whole-body) and with physiological stress such as exercise (focal). Hypoxemia is a recognized key feature of respiratory dysfunction presenting as a consequence of impaired gas exchange in acute and chronic respiratory disease. Episodic hypoxemia is a cardinal feature of respiratory control disorders such as OSAS, a very common primary syndrome, ${ }^{28}$ which can also present in hypoxemic patients with COPD, that is, overlap syndrome. ${ }^{29}$ Often underappreciated, hypoxemia also presents in neurodegenerative diseases such as amyotrophic lateral sclerosis, ${ }^{30}$ Parkinson's disease, ${ }^{31}$ and Alzheimer's disease, ${ }^{32}$ as well as in neuromuscular conditions such as muscular dystrophy, ${ }^{33}$ often culminating in end-stage respiratory failure.

More than merely a symptom of respiratory malady, hypoxia is a potent stimulus driving phenotypic change in many systems, including the cardiorespiratory control system. Empirically, hypoxia is a major driver of respiratory system plasticity (Table 1). This includes potential for adaptive or compensatory plasticity in respiratory muscle that serves to protect against the challenge of oxygen deficit as well as the contemporaneous capacity to drive aberrant remodeling, which can further exacerbate and perpetuate respiratory dysfunction. ${ }^{27,34,35}$

Of importance, but beyond the scope of this review, there is tremendous scope for hypoxia-dependent signaling to alter neural regulation of respiratory control through actions on sensory, central integrative, and motor systems that could exacerbate or compensate for muscle-specific changes. ${ }^{35}$ Outcomes are dependent upon, inter alia, the context of hypoxic exposure (e.g., age, sex, co-stimuli, comorbidity), the explicit nature of the hypoxia stimulus (e.g., pattern, intensity, and 
Table I Effects of hypoxic exposure on respiratory muscle form and function

\begin{tabular}{|c|c|c|}
\hline Phenotype & Intermittent hypoxia & Sustained hypoxia \\
\hline Disease; environment & Sleep-disordered breathing & Lung diseases; altitude \\
\hline Muscle force & Weakness $^{15,17,26,36,37,39,46,55}$ & Unchanged ${ }^{7,72,73} ;$ weakness $^{7,72,75,77}$ \\
\hline Muscle endurance & $\begin{array}{l}\text { Mixed effects: unchanged }{ }^{15,17,46,47} ; \text { increased }^{52} \text {; } \\
\text { decreased }^{37,40-42,44,45,50}\end{array}$ & Mixed effects: unchanged ${ }^{14,73} ;$ increased $^{7} ;$ decreased $^{14,75,77}$ \\
\hline Muscle CSA & Unchanged ${ }^{46,47} ;$ decreased ${ }^{15,52}$ & Unchanged or decreased: muscle specific ${ }^{7,14,73}$ \\
\hline Muscle fibers & $\begin{array}{l}\text { Mixed effects: no change }{ }^{15,47,57} \text {; increased density } \\
\text { of type } 1^{52} ; \text { increased density of type } 2^{37,39,40,50,53}\end{array}$ & Increased density of type $I^{7}$; slow-to-fast ${ }^{80}$ \\
\hline Duration-dependent & Weeks $>$ days $^{50}$ & Weeks $>$ days s,75,77 $^{7}$ \\
\hline Acute effects & Yes (hours-to-days) ${ }^{26,39,52}$ & Yes (hours) ${ }^{78,79}$ \\
\hline Intensity-dependent & $Y_{e s^{50}}$ & Yes (limited data) $)^{7,72 * *}$ \\
\hline Age-dependent & Young $>$ adult $>$ old $15,17,46,47,57$ & Young $>$ adult ${ }^{14}$ \\
\hline Sex-dependent & Males $>$ females ${ }^{46} ;$ OVX $>$ control $^{40,59}$ & Unknown: sex difference in acute hypoxia ${ }^{81}$ \\
\hline Redox-dependent & $\begin{array}{l}\text { Markers of oxidative stress }{ }^{50,52,88} \text {; protein oxidation }{ }^{52} \text {; } \\
\text { dysfunction without protein oxidation or lipid } \\
\text { peroxidation }\end{array}$ & Time-dependent protein oxidation ${ }^{75,77}$ \\
\hline HIF-I $\alpha$ stabilization & Mixed $^{41,42,44,82}$ & Muscle-specific ${ }^{75,77}$ \\
\hline Mitochondrial remodeling & Autophagy $^{52} ;$ dysfunction ${ }^{45,85,87}$ & Decreased density; increased efficiency ${ }^{73,89}$ \\
\hline Metabolic remodeling & Modest $^{15,17,46,47,82}$ & Extensive $^{75,77}$ \\
\hline Hypoxic tolerance & Muscle specific: unchanged ${ }^{46,47} ;$ improved $^{51}$ & Improved ${ }^{76}$ \\
\hline
\end{tabular}

Notes: Summary of the effects of chronic intermittent hypoxia and chronic sustained hypoxia on respiratory muscle form and function derived from studies in rodent models. *Inferred from reference 72 compared with reference 7.

Abbreviations: CSA, cross-sectional area; HIF-I $\alpha$, hypoxia inducible factor-I alpha; OVX, ovariectomized.

duration of exposure), and the inherent or acquired capacity to tolerate and/or recover from hypoxic insult.

\section{$\mathrm{ClH}$ modeling human respiratory disease causes respiratory muscle dysfunction}

Several groups have independently observed upper airway dilator and diaphragm muscle weakness and/or fatigue following exposure to $\mathrm{CIH}$ in animal models modeling the $\mathrm{CIH}$ that occurs in human OSAS. ${ }^{15-17,26,36-46} \mathrm{CIH}$-induced upper airway muscle dysfunction does not require structural remodeling in the form of muscle fiber atrophy and/or fibertype transitions, ${ }^{17,47}$ although fiber-type transitions including increased expression of MHC type 2B fast fibers have been described. ${ }^{37,39,40}$ This is of interest given that slow-to-fast fiber transitions are reported in human OSAS ${ }^{11,12,48}$ and that the relative area of fast fatigable fibers is increased in the English bulldog model of OSAS. ${ }^{49}$ The duration of CIH exposure is a key determinant of the respiratory muscle responses ${ }^{39,50}$ such that several weeks of exposure increases upper airway muscle fatigue $^{37,40-42,44,45}$ and impairs recovery from fatigue. ${ }^{36,37}$

Less is known about the effects of $\mathrm{CIH}$ on diaphragm muscle form and function. Short-term exposure to IH (hours to days) has been reported to have no deleterious effect on diaphragm structure and contractile function in rat. ${ }^{39,51}$ However, a subsequent study convincingly demonstrated that IH exposure of just 4 days in mice was sufficient to cause autophagyassociated atrophy of diaphragm, leading to weakness, which recovered in normoxia. ${ }^{52}$ Interestingly, Shortt et al ${ }^{50}$ demon- strated that rat diaphragm force and fatigue are affected in a manner dependent on the duration and intensity of the $\mathrm{CIH}$ exposure. Weakness and increased fatigue were observed following a 2-week exposure to IH (fractional of inspired oxygen concentration $\left[\mathrm{FiO}_{2}\right]=5 \%$ at nadir; 20 cycles per hour; 8 hours per day), with no muscle atrophy, but an increase in the relative area of MHC type 2B fast fibers and concomitant increase in the relative area of sarcoplasmic reticulum calcium ATPase 1-expressing fibers. ${ }^{50,53}$ This is consistent with the slow-to-fast transitions in rat airway dilator muscle. ${ }^{37,39}$ Longer durations of $\mathrm{CIH}\left(\mathrm{FiO}_{2}=6 \%-8 \%\right.$ at nadir; 120 cycles per hour; 8 hours per day for 5 weeks) are associated with increased fatigue and impaired recovery from fatigue in rat diaphragm. ${ }^{54}$ Moreover, exposure to $\mathrm{CIH}$ exacerbates diaphragm muscle weakness in $\mathrm{mdx}$ dystrophic mice, ${ }^{55}$ although it remains to be determined if there is altered susceptibility to CIH stress in mdx respiratory muscle. This is important to determine given that sleepdisordered breathing is a feature of muscular dystrophy. ${ }^{56}$

Furthermore, there is growing evidence to suggest that the effects of exposure to $\mathrm{CIH}$ on respiratory muscle performance may be age- and sex dependent. Upper airway dilator muscle weakness is observed following $\mathrm{CIH}$ exposure $\left(\mathrm{FiO}_{2}=5 \%\right.$ at nadir; 20 cycles per hour; 8 hours per day) in young adult male rats, ${ }^{46}$ but not in middle-aged male rats, ${ }^{47}$ consistent with the findings of others who reported no structural or functional change in the upper airway muscles following $\mathrm{CIH}$ exposure in old-aged rats. ${ }^{57}$ In contrast, increased susceptibility to upper airway muscle weakness following $\mathrm{CIH}\left(\mathrm{FiO}_{2}=5 \%\right.$ at 
nadir; 12 cycles per hour; 8 hours per day) is observed during early life compared with adult animals. ${ }^{17,58}$ Moreover, upper airway muscle dysfunction following neonatal exposure to $\mathrm{CIH}$ extends into young adulthood suggesting lasting effects of antecedent exposure to $\mathrm{CIH}$ during critical windows of development. ${ }^{15,17}$ Indeed, neonatal exposure to $\mathrm{CIH}$ primes increased susceptibility to subsequent hypoxic stress in later life. ${ }^{15}$ Unlike airway dilator muscle responses, ${ }^{15,17}$ exposure to $\mathrm{CIH}$ ( $\mathrm{FiO}_{2}=5 \%$ at nadir; 12 cycles per hour; 8 hours per day) has no discernible effect on diaphragm form or function, ${ }^{16}$ an outcome that may relate to intrinsic differences in upper airway and diaphragm muscle fiber type and metabolic programming during development. ${ }^{13-17}$

That sex hormones may influence respiratory muscle responses to intermittent hypoxic stress is evinced by studies involving male vs. female comparison, ${ }^{40,46,59,60}$ ovariectomized females, ${ }^{40,59}$ estrogen replacement therapy, ${ }^{40,41}$ and phytoestrogens. ${ }^{42,44,59,61}$ Female skeletal muscle is usually less fatigable than male, but differences are task specific and dependent upon contraction velocity and specific muscle groups, ${ }^{62,63}$ though of interest, sex differences can persist with aging. ${ }^{64}$ Estrogen-dependent signaling may confer protection in upper airway muscles in response to $\mathrm{CIH}$, which may explain in part the recognized 2-3-fold lower incidence of OSAS in premenopausal women compared with men. ${ }^{65-68} \mathrm{~A}$ recent review of the role of sex in sleep apnea is provided by O'Halloran et al. ${ }^{27}$

The significance of these findings is that upper airway $^{11,12,48,69}$ and diaphragm muscle dysfunction ${ }^{70,71}$ is reported in human OSAS, and myogenic mechanisms may contribute to the pathophysiology of the disorder. ${ }^{26,27,69}$ Although it is recognized that OSAS is a complex multifaceted condition, exposure to $\mathrm{CIH}$ because of recurrent pauses in ventilation is a major pathogenic factor driving multisystem morbidity, including aberrant function in the effector muscles of breathing. ${ }^{27,35}$ An understanding of the mechanisms by which $\mathrm{CIH}$ drives respiratory muscle dysfunction offers potential for the development of therapeutic strategies serving to protect respiratory muscle performance.

\section{$\mathrm{CSH}$ modeling human respiratory disease causes respiratory muscle dysfunction}

Exposure to sustained hypoxia is a feature of altitude and respiratory disease. Exposure to hypobaric hypoxia (380 $\mathrm{mmHg}$, equivalent to $\sim 10 \%$ inspired oxygen) for 6 weeks during adulthood has differential effects on rat upper airway dilator and diaphragm muscle endurance, ${ }^{7,72}$ with evidence that diaphragm muscle endurance is preserved ${ }^{72,73}$ or enhanced, ${ }^{7}$ whereas upper airway dilator muscle endurance is preserved ${ }^{7}$ or decreased..$^{72}$ Exposure to $10 \%$ inspired oxygen for 4 weeks in mice decreased diaphragm muscle fiber cross-sectional areas and improved mitochondrial aerobic efficiency, likely contributing to the maintenance of diaphragm endurance. ${ }^{73}$ $\mathrm{CSH}$ decreasing diaphragm muscle fiber cross-sectional areas in rat $^{7,74}$ and mouse ${ }^{73}$ could be viewed as advantageous in decreasing oxygen diffusion distance. ${ }^{34,73}$ On the other hand, evidence points to consequential time-dependent and substantial loss of force-generating capacity, ${ }^{7,75}$ which is also consistent with decreased fiber cross-sectional areas. ${ }^{7,73}$ Physiological tradeoffs may be at play in response to hypoxia as rat diaphragm shows evidence of increased hypoxic tolerance compared with normoxic diaphragm following $\mathrm{CSH}^{76}$ and $\mathrm{CIH}$ exposure. ${ }^{51}$

Exposure of mice to $10 \%$ inspired oxygen for 6 weeks resulted in significant upper airway dilator ${ }^{77}$ and diaphragm weakness $^{75}$ because of time-dependent progressive oxidation of proteins key to muscle contraction, metabolism, and cellular homeostasis. ${ }^{34,75,77}$ Remarkably, recent studies have demonstrated that just 6-8 hours of exposure to sustained hypoxia in mice is sufficient to cause upper airway dilator ${ }^{78}$ and diaphragm muscle weakness. ${ }^{79}$ Of interest, mdx dystrophic upper airway dilator muscle, which is weak compared with wild-type muscle, shows evidence of hypoxic tolerance suggesting a potential role for hypoxia-dependent signaling in mdx muscle dysfunction. ${ }^{78}$ This is interesting in the context of Duchenne muscular dystrophy given that hypoxemia is a feature of the debilitating neuromuscular disorder owing to respiratory insufficiency. ${ }^{33,56}$

Exposure to sustained hypoxia for 60 days during early life had no effect on diaphragm myosin fiber complement, whereas exposure for 7-9 months caused a slow-to-fast myosin transition. ${ }^{80}$ Exposure to sustained hypoxia (450 $\mathrm{mmHg}$, equivalent to $\sim 12 \%$ inspired oxygen) for 7 days during neonatal development causes rat airway dilator muscle dysfunction that is age-dependent, waning with advancing age beyond the neonatal period, but persisting during normoxic recovery for several weeks if exposure to sustained hypoxia occurred in the first or second week of life. ${ }^{14}$ Sustained hypoxia did not affect muscle fiber-type distribution, or the activity of key representative oxidative or glycolytic enzyme activities. ${ }^{14}$ Similar to observations following exposure to $\mathrm{CIH}$ during early life, ${ }^{16}$ sustained hypoxia for 7 days had no deleterious effect on rat diaphragm functional properties ${ }^{14}$ revealing muscle-specific effects of sustained hypoxic stress, with relative resilience in diaphragm muscle compared with airway dilator muscle to $\mathrm{CSH}$ during early development. 
A recent study revealed sex differences intrinsic to respiratory muscle in response to severe hypoxia, ${ }^{81}$ but otherwise little is known concerning putative sex differences in respiratory muscle responses to $\mathrm{CSH}$ representing a significant gap in our knowledge worthy of further investigation.

Respiratory muscle remodeling in response to short-term hypoxic stress has relevance to respiratory conditions such as acute respiratory distress syndrome, particularly in the light of evidence that diaphragm weakness is a major predictor of patient morbidity and clinical outcome. ${ }^{24}$

\section{Redox stress is a major driver of respiratory muscle dysfunction}

Altered redox signaling is implicated in both $\mathrm{CSH}-$ and $\mathrm{CIH}-$ induced respiratory muscle dysfunction albeit differentially. Williams et al ${ }^{82}$ reported that exposure to $\mathrm{CIH}$ caused a significant increase in upper airway muscle nicotinamide adenine dinucleotide phosphate (NADPH) oxidase expression, although airway dilator muscle weakness presents in the absence of overt muscle damage or oxidative stress, revealing that altered redox signaling is sufficient to drive dysfunction. ${ }^{17,50,82}$ It is clear that reactive oxygen species (ROS) inhibit upper airway dilator muscle function, ${ }^{83,84}$ although relatively modest oxidative stress is evoked in respiratory muscle during $\mathrm{CIH}$ exposure evidenced by decreased aconitase and glutathione reductase activities without protein oxidation or lipid peroxidation. ${ }^{82}$ This has led to speculation that $\mathrm{CIH}$ enhances NADPH oxidase superoxide production in myocellular microdomains, tonically suppressing force-generating capacity of respiratory muscles. ${ }^{35,82}$ This hypothesis is supported by the observation that acute antioxidant administration is capable of reversing $\mathrm{CIH}$-induced airway dilator muscle weakness ${ }^{46}$ and that the phytoestrogen genistein may improve upper airway muscle function through antioxidant mechanisms after $\mathrm{CIH}$ insult. ${ }^{42}$ Thus, enhanced redox-dependent suppression of force may be sufficient to drive $\mathrm{CIH}$-induced respiratory muscle weakness. Longer exposure to $\mathrm{CIH}$ increases endoplasmic reticulum stress and apoptotic signaling in upper airway muscles, ${ }^{85}$ and causes mitochondrial remodeling and dysfunction ${ }^{45,86,87}$ for which altered redox homeostasis could be causal and/or a consequence.

In mouse diaphragm, 4 days of IH exposure increased autophagy-dependent protein degradation causing muscle weakness with increased antioxidant gene expression. ${ }^{52} \mathrm{~A}$ modest $\mathrm{CIH}$ exposure over 2 weeks increased rat diaphragm pro-inflammatory cytokines and superoxide anion generation, ${ }^{88}$ and rat diaphragm dysfunction following $\mathrm{CIH}$ exposure is associated with modest attendant oxidative stress. ${ }^{50}$

In stark contrast to $\mathrm{CIH}$ models, a pronounced timedependent redox stress characterizes functional impairments in mouse upper airway ${ }^{77}$ and diaphragm ${ }^{34,75}$ muscles in response to CSH stress. Redox modulation of upper airway muscle metabolism and function, which appears hypoxia inducible factor-1 alpha (HIF-1 $\alpha$ )-independent, ${ }^{77}$ gives rise to pronounced muscle weakness without proteolytic degradation, notwithstanding the substantial temporal increase in muscle protein carbonyl content, which, as revealed by twodimensional proteomic analysis, ranges from mitochondria to the cross-bridges. ${ }^{77}$ Unlike diaphragm, fast fiber airway dilator muscle cross-sectional area is spared in CSH, and indeed hypertrophy has been reported in chronic hypoxic rat sternohyoid muscle, ${ }^{7}$ with evidence too of increased sternohyoid force-generating capacity in response to hypobaric hypoxia ( $450 \mathrm{mmHg}$, equivalent to $\sim 12 \%$ inspired oxygen). ${ }^{72}$ More intense hypoxia over the same period (10\% inspired oxygen for 6 weeks) does not affect rat upper airway muscle force, ${ }^{7}$ but causes airway dilator muscle weakness in mice, which appears related to direct redox modulation of the contractile apparatus. ${ }^{77}$

There is pronounced diaphragm muscle protein carbonylation during exposure to $\mathrm{CSH} .{ }^{75}$ In mouse diaphragm, CSH decreased mitochondrial density, ${ }^{89}$ inhibited mTORdependent protein synthesis, and promoted FOXO-3a and 20S proteasome activity. ${ }^{75}$ Pronounced metabolic remodeling occurs in CSH mouse diaphragm, which may be partly HIF-1 $\alpha$-dependent and thus redox-dependent, ${ }^{75}$ with altered mitochondrial respiration rates, ${ }^{73}$ decreased oxidative and glycolytic enzyme activities, and an apparent increased reliance on fatty acid metabolism. ${ }^{75}$ The redox-dependent plasticity confers early adaptive responses, ${ }^{7,73,75,89}$ which culminate in time-dependent diaphragm weakness, perhaps a tradeoff for cellular adaptive responses improving hypoxic tolerance. ${ }^{34,75}$ Lewis and O'Halloran ${ }^{34}$ have recently provided a review of redox-dependent adaptive and maladaptive responses of diaphragm muscle to $\mathrm{CSH}$ with relevance to respiratory muscle performance.

\section{Antioxidant intervention prevents hypoxia-dependent respiratory muscle dysfunction}

Antioxidant supplementation can ameliorate or prevent CIHinduced respiratory muscle dysfunction..$^{38,46,50}$ The endogenous antioxidant, glutathione, appears pivotal to respiratory 
muscle responses to $\mathrm{CIH}$. Pharmacological depletion of glutathione exacerbates $\mathrm{CIH}$-induced airway dilator muscle fatigue, ${ }^{38}$ whereas $\mathrm{N}$-acetyl cysteine (NAC) supplementation, which boosts endogenous glutathione levels, is the most effective intervention in preventing $\mathrm{CIH}$-induced diaphragm weakness and fatigue, ${ }^{50}$ although this may also relate to general antioxidant effects of the supplement. Phytoestrogens are effective in preventing $\mathrm{CIH}$-induced airway dilator muscle dysfunction with genistein demonstrating redox effects. . $^{42,4}$ There may be antioxidant effects of female sex hormones given that estrogen enhances cellular antioxidant defense. ${ }^{90}$ Antioxidant intervention is highly effective in preventing CSH-induced airway dilator ${ }^{77}$ and diaphragm dysfunction. ${ }^{75}$ Chronic antioxidant supplementation prevented respiratory muscle protein carbonylation following 6 weeks of sustained hypoxia in mice. ${ }^{75,77} \mathrm{NAC}$ was especially effective in preventing respiratory muscle force decline following exposure to $\mathrm{CSH}^{75,77}$ A pivotal role of ROS in driving maladaptive responses in chronic hypoxic mouse diaphragm is further revealed by antioxidant suppression of p38 MAP kinase, ${ }^{75}$ and likely subsequent suppression of FOXO-3a-dependent atrophy and proteolytic degradation. ${ }^{91}$ In this way it appears that NAC supplementation prevents CSH-induced diaphragm weakness. A comparison of the effects of antioxidants on muscle function in animal models of hypoxic stress is presented in Table 2. The data make a compelling argument in favor of exploration of the potential benefits of antioxidant intervention in human respiratory patients to limit or prevent respiratory muscle dysfunction arising from hypoxic stress, whatever the pattern of exposure (Figure 1).

\section{Activity-induced respiratory muscle plasticity: does muscle recruitment during hypoxic stress promote or mitigate respiratory muscle dysfunction?}

It is important to acknowledge that respiratory muscle activation is likely a critical co-factor, in addition to direct hypoxic stress, driving phenotypic change in muscle in response to exposure to hypoxia. It is well appreciated that respiratory muscle recruitment is altered in respiratory disease states. In OSAS, periodic occlusion of the upper airway during

Table 2 Effects of antioxidants on hypoxic respiratory muscle form and function

\begin{tabular}{|c|c|c|}
\hline Phenotype & Intermittent hypoxia & Sustained hypoxia \\
\hline \multirow[t]{4}{*}{ Muscle force } & UA muscle improvement - Tempol ${ }^{46}$ & UA muscle improvement - NAC ${ }^{77}$ \\
\hline & Diaphragm muscle improvement - NAC ${ }^{50}$ & *UA muscle no change - Tempol ${ }^{77}$ \\
\hline & & Diaphragm muscle improvement $-\mathrm{NAC}^{75}$ \\
\hline & & Diaphragm muscle no change - Tempol ${ }^{75}$ \\
\hline \multirow[t]{5}{*}{ Muscle endurance } & UA muscle improvement - Genistein ${ }^{42}$ & *UA muscle no change - Tempol ${ }^{77}$ \\
\hline & UA muscle improvement - NAC 38 & *UA muscle no change - NAC 77 \\
\hline & Diaphragm muscle improvement - NAC ${ }^{50}$ & Diaphragm muscle improvement $-\mathrm{NAC}^{75}$ \\
\hline & Diaphragm muscle improvement - Tempol $^{50}$ & Diaphragm muscle no change - Tempol $^{75}$ \\
\hline & Diaphragm muscle improvement - Apocynin ${ }^{50}$ & \\
\hline \multirow[t]{3}{*}{ Protein signaling } & UA muscle increased $\mathrm{p}$-ERKI/2/ERKI/2 content - & UA muscle unchanged phospho-JNK - Tempol ${ }^{77}$ \\
\hline & Genistein $^{42}$ & UA muscle unchanged phospho-JNK - NAC 77 \\
\hline & & Diaphragm muscle decreased phospho-p38 - NAC ${ }^{75}$ \\
\hline Antioxidant activity & UA muscle increased SOD, GPx, and catalase - Genistein ${ }^{42}$ & - \\
\hline \multirow[t]{2}{*}{ HIF-I $\alpha$ stabilization } & - & Diaphragm muscle decreased HIF-I $\alpha-\mathrm{NAC}^{75}$ \\
\hline & & Diaphragm muscle decreased HIF-I $\alpha-$ Tempol $^{75}$ \\
\hline
\end{tabular}

Mitochondria

UA muscle decreased mitochondrial ROS - Genistein ${ }^{42}$

Oxidative stress

UA muscle decreased MDA - Genistein ${ }^{42}$ Diaphragm muscle decreased GSSG:GSH - NAC ${ }^{50}$

UA muscle decreased carbonyl content - Tempol
UA muscle decreased carbonyl content - NAC ${ }^{77}$
UA muscle increased muscle free thiol content - Tempol $^{77}$
UA muscle increased muscle free thiol content - NAC $^{77}$
Diaphragm muscle decreased carbonyl content - Tempol $^{75}$
Diaphragm muscle decreased carbonyl content - NAC $^{75}$
Diaphragm muscle increased free thiol content - Tempol $^{75}$
Diaphragm muscle increased free thiol content - NAC $^{75}$
Diaphragm muscle decreased fatigue tolerance - L-NNA $^{76}$

Hypoxic tolerance

$-$

Notes: Summary of the effects of antioxidants on hypoxic respiratory muscle form and function derived from studies in rodent models. *Improved but not statistically significant.

Abbreviations: GPx, glutathione peroxidase; GSH, glutathione; GSSG, glutathione disulfide; HIF-I $\alpha$, hypoxia inducible factor-Ialpha; L-NNA, NG-nitro-L-arginine (a competitive inhibitor of nitric oxide synthase); MDA, malondialdehyde; NAC, N-acetyl cysteine; p-ERKI/2, phosphorylated extracellular signal-related kinases; phosphorJNK, phosphorylated c-Jun N-terminal kinases; ROS, reactive oxygen species; SOD, superoxide dismutase; UA, upper airway. 


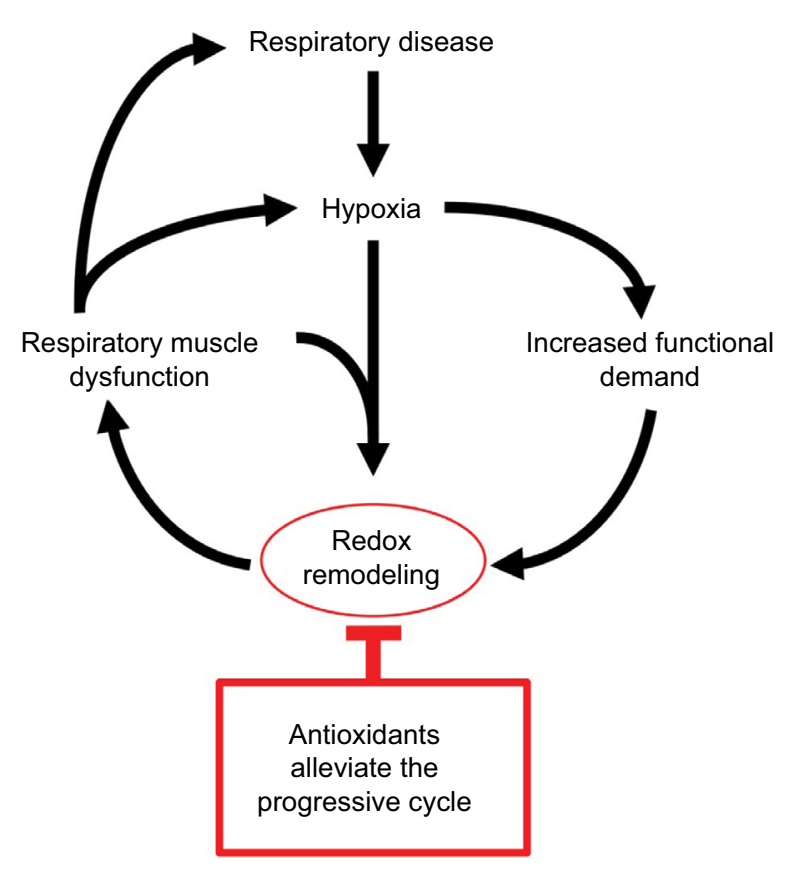

Figure I Hypoxia-dependent respiratory muscle dysfunction.

Notes: Redox stress is pivotal to hypoxia-dependent respiratory muscle structural-, functional-, and metabolic remodeling. Antioxidant intervention interrupts the vicious feedback cycle that perpetuates respiratory muscle dysfunction. The potential benefits of antioxidants in ameliorating respiratory muscle dysfunction in human hypoxic disease warrant investigation.

sleep results in loading of the upper airway muscles ${ }^{69}$ and progressive recruitment of the diaphragm and accessory inspiratory muscles ${ }^{71}$ owing to considerable increases in ventilatory drive. Thus, mechanical as well as chemical stimuli are key determinants of respiratory muscle activity in OSAS. ${ }^{6}$ In hypoxic respiratory diseases such as COPD, airway narrowing increases the load on respiratory muscles, ${ }^{92}$ and hyperinflation can further disadvantage the diaphragm because of altered length-tension relationships. ${ }^{93,94}$ These multifaceted, complex changes make it extremely difficult to assess the independent influences of various stressors on respiratory muscle performance in disease..$^{95}$

With regard to animal models of hypoxic disease, mechanical confounders are largely eliminated. However, it is important to recognize that hypoxic stress produces an integrated cardiorespiratory response that results in increased activation of the respiratory muscles and attendant increases in respiratory muscle blood flow to support hypoxic hyperventilation. Thus, hypoxia results in a multimodal stimulus at the level of the respiratory muscles. Intense exercise can cause diaphragm fatigue, ${ }^{96,97}$ which is exacerbated by acute hypoxia, ${ }^{98-101}$ with implications for respiratory sensations (dyspnea) in patients. ${ }^{102}$ As ROS are generated in exercising muscle, ${ }^{103,104}$ it is conceivable that increased respiratory muscle work, particularly in the face of accompanying hypoxic stress, is an additional stressor to respiratory muscle in chronic models of hypoxia. We reason that this may be especially likely in $\mathrm{CSH}$, which necessitates a persistent hyperventilation during chronic exposure. By its nature, $\mathrm{CIH}$ presents an intermittent activation of the respiratory muscles, though persistent hyperventilation following exposure to $\mathrm{CIH}$ has also been described. ${ }^{105}$ In short, though beyond the scope of this review, we acknowledge that increased respiratory muscle work during hypoxic exposure may contribute additional redox stress in respiratory muscles.

Notwithstanding the potential for activity-induced aberrant redox remodeling in respiratory muscles, which could exacerbate respiratory muscle performance by enhancing muscle oxidative stress, it is also plausible that activityinduced plasticity in respiratory muscles could drive adaptive outcomes. It has been shown that aerobic exercise results in dramatic adaptive changes in the diaphragm proteome. ${ }^{106}$ Exercise leads to improved diaphragm ${ }^{107,108}$ and upper airway ${ }^{109}$ muscle endurance, resulting from increased aerobic capacity. Increased endurance ${ }^{7}$ and mitochondrial efficiency ${ }^{73,89}$ in diaphragm, which differs to responses in limb muscles, ${ }^{73,110,111}$ might relate to differences in activation patterns as well as intrinsic differences in muscle form. Thus, one could argue that activity-induced plasticity might limit the deleterious effects of hypoxic stress. It should be understood that this will vary within and between diseases. For example, exercise worsens dystrophic diaphragm function $^{112}$ purportedly because of increased mechanical stress on fragile myofibers. Collectively, it is difficult to discern the dynamic interplay between hypoxia, muscle activity (exercise), and cumulative redox balance in muscle, but these complex interactions are likely pivotal determinants of respiratory muscle performance in chronic disease. An integrated portfolio of stimuli must be borne in mind when considering the effects of hypoxia on respiratory muscle physiology (Figure 1), which likely result in dynamic temporospatial $\mathrm{PO}_{2}$ gradients in muscle.

\section{Conclusion and perspective}

Despite the clinical relevance, there is a relative paucity of information on hypoxic adaptation in respiratory muscles. Recent studies in animal models illustrate that hypoxia, modeling human respiratory diseases, is a potent stimulus provoking plastic changes in respiratory muscles with implications for physiological function (Table 1). Redox stress is pivotal to hypoxic-dependent respiratory muscle dysfunction manifesting in pronounced weakness and fatigue, evidenced by redox remodeling (Table 1) and beneficial effects of antioxidant 
supplementation (Table 2). The data reviewed herein suggest that greater appreciation of hypoxia-dependent remodeling in respiratory muscle is required to better inform mechanistic insight of respiratory disease and to shape interventional studies to halt or reverse disease progression. Hypoxia emerges as a likely major player in respiratory muscle remodeling in pulmonary diseases and respiratory control disorders. Antioxidants, in particular NAC, have proven highly effective in animal models of hypoxia, strengthening the case for their potential use in the treatment of respiratory morbidity in humans. The animal studies provide an evidence base in strong support of the need to explore adjunctive antioxidant therapies for muscle dysfunction in human respiratory disease. Beyond the relevance to primary respiratory diseases, we further suggest that hypoxia-dependent respiratory system maladaptation is an area worthy of careful consideration in neurodegenerative and neuromuscular conditions, both in terms of disease progression and interventional strategies.

\section{Acknowledgments}

Studies from the laboratory of $\mathrm{KDOH}$ were funded by the Health Research Board (Ireland), The Irish Research Council, and were partly supported by the University College Dublin School of Medicine and University College Cork, Department of Physiology, and the University College Cork Strategic Research Fund.

\section{Disclosure}

The authors report no conflicts of interest in this work.

\section{References}

1. Goldman MD, Grassino A, Mead J, Sears TA. Mechanics of the human diaphragm during voluntary contraction: dynamics. J Appl Physiol Respir Environ Exerc Physiol. 1978;44(6):840-848.

2. Roussos C, Macklem PT. The respiratory muscles. $N$ Engl J Med. 1982;307(13):786-797.

3. De Troyer A, Estenne M. Functional anatomy of the respiratory muscles. Clin Chest Med. 1988;9(2):175-193.

4. De Troyer A, Boriek AM. Mechanics of the respiratory muscles. Compr Physiol. 2011;1(3):1273-1300.

5. White DP. Pathogenesis of obstructive and central sleep apnea. Am J Respir Crit Care Med. 2005;172(11):1363-1370.

6. White DP, Younes MK. Obstructive sleep apnea. Compr Physiol. 2012;2(4):2541-2594.

7. McMorrow C, Fredsted A, Carberry J, et al. Chronic hypoxia increases rat diaphragm muscle endurance and sodium-potassium ATPase pump content. Eur Respir J. 2011;37(6):1474-1481.

8. Meznaric M, Cvetko E. Size and proportions of slow-twitch and fasttwitch muscle fibers in human costal diaphragm. Biomed Res Int. 2016;2016:5946520.

9. Seven YB, Mantilla CB, Sieck GC. Recruitment of rat diaphragm motor units across motor behaviors with different levels of diaphragm activation. J Appl Physiol (1985). 2014;117(11):1308-1316.

10. Greising SM, Mantilla CB, Sieck GC. Functional measurement of respiratory muscle motor behaviors using transdiaphragmatic pressure. Methods Mol Biol. 2016;1460:309-319.
11. Series F, Cote C, Simoneau JA, et al. Physiologic, metabolic, and muscle fiber type characteristics of musculus uvulae in sleep apnea hypopnea syndrome and in snorers. J Clin Invest. 1995;95(1): 20-25.

12. Series FJ, Simoneau SA, St Pierre S, Marc I. Characteristics of the genioglossus and musculus uvulae in sleep apnea hypopnea syndrome and in snorers. Am J Respir Crit Care Med. 1996;153(6 Pt 1): 1870-1874.

13. O'Connell RA, Carberry J, O'Halloran KD. Sternohyoid and diaphragm muscle form and function during postnatal development in the rat. Exp Physiol. 2013;98(9):1386-1400.

14. Carberry JC, McMorrow C, Bradford A, Jones JF, O’Halloran KD. Effects of sustained hypoxia on sternohyoid and diaphragm muscle during development. Eur Respir J. 2014;43(4):1149-1158.

15. McDonald FB, Dempsey EM, O’Halloran KD. Early Life exposure to chronic intermittent hypoxia primes increased susceptibility to hypoxia-induced weakness in rat sternohyoid muscle during adulthood. Front Physiol. 2016;7:69.

16. McDonald FB, Dempsey EM, O'Halloran KD. Effects of gestational and postnatal exposure to chronic intermittent hypoxia on diaphragm muscle contractile function in the rat. Front Physiol. 2016;7:276.

17. McDonald FB, Williams R, Sheehan D, O’Halloran KD. Early life exposure to chronic intermittent hypoxia causes upper airway dilator muscle weakness, which persists into young adulthood. Exp Physiol. 2015;100(8):947-966.

18. Polla B, D’Antona G, Bottinelli R, Reggiani C. Respiratory muscle fibres: specialisation and plasticity. Thorax. 2004;59(9):808-817.

19. Jiao GY, Hao LY, Chen L, et al. High levels of positive end-expiratory pressure preserve diaphragmatic contractility during acute respiratory distress syndrome in rats. Exp Physiol. 2015;100(8):967-976.

20. Li LF, Chang YL, Chen NH, et al. Inhibition of Src and forkhead box $\mathrm{O} 1$ signaling by induced pluripotent stem-cell therapy attenuates hyperoxia-augmented ventilator-induced diaphragm dysfunction. Transl Res. 2016;173:131-147. e1.

21. Hooijman PE, Beishuizen A, Witt CC, et al. Diaphragm muscle fiber weakness and ubiquitin-proteasome activation in critically ill patients. Am J Respir Crit Care Med. 2015;191(10):1126-1138.

22. Hudson MB, Smuder AJ, Nelson WB, et al. Partial support ventilation and mitochondrial-targeted antioxidants protect against ventilatorinduced decreases in diaphragm muscle protein synthesis. PLoS One. 2015;10(9):e0137693.

23. Barreiro E, Gea J. Respiratory and limb muscle dysfunction in COPD. COPD. 2015;12(4):413-426.

24. Supinski GS, Callahan LA. Diaphragm weakness in mechanically ventilated critically ill patients. Crit Care. 2013;17(3):R120.

25. Petrof BJ, Hendricks JC, Pack AI. Does upper airway muscle injury trigger a vicious cycle in obstructive sleep apnea? A hypothesis. Sleep. 1996;19(6):465-471.

26. Bradford A, McGuire M, O’Halloran KD. Does episodic hypoxia affect upper airway dilator muscle function? Implications for the pathophysiology of obstructive sleep apnoea. Respir Physiol Neurobiol. 2005;147(2-3):223-234.

27. O'Halloran KD, Lewis P, McDonald F. Sex, stress and sleep apnoea: decreased susceptibility to upper airway muscle dysfunction following intermittent hypoxia in females. Respir Physiol Neurobiol. Epub 2016 Nov 21.

28. Young T, Evans L, Finn L, Palta M. Estimation of the clinically diagnosed proportion of sleep apnea syndrome in middle-aged men and women. Sleep. 1997;20(9):705-706.

29. McNicholas WT. Chronic obstructive pulmonary disease and obstructive sleep apnoea-the overlap syndrome. J Thorac Dis. 2016;8(2): 236-242.

30. Park SY, Kim SM, Sung JJ, et al. Nocturnal hypoxia in ALS is related to cognitive dysfunction and can occur as clusters of desaturations. PLoS One. 2013;8(9):e75324.

31. Serebrovskaya T, Karaban I, Mankovskaya I, Bernardi L, Passino C, Appenzeller O. Hypoxic ventilatory responses and gas exchange in patients with parkinson's disease. Respiration. 1998;65(1):28-33. 
32. Peers C, Pearson HA, Boyle JP. Hypoxia and Alzheimer's disease. Essays Biochem. 2007;43:153-164.

33. Bersanini C, Khirani S, Ramirez A, et al. Nocturnal hypoxaemia and hypercapnia in children with neuromuscular disorders. Eur Respir J. 2012;39(5):1206-1212.

34. Lewis P, O'Halloran KD. Diaphragm muscle adaptation to sustained hypoxia: lessons from animal models with relevance to high altitude and chronic respiratory diseases. Front Physiol. 2016;7:623.

35. O'Halloran KD. Chronic intermittent hypoxia creates the perfect storm with calamitous consequences for respiratory control. Respir Physiol Neurobiol. 2016;226:63-67.

36. McGuire M, MacDermott M, Bradford A. Effects of chronic episodic hypoxia on rat upper airway muscle contractile properties and fibertype distribution. Chest. 2002;122(3):1012-1017.

37. McGuire M, MacDermott M, Bradford A. The effects of chronic episodic hypercapnic hypoxia on rat upper airway muscle contractile properties and fiber-type distribution. Chest. 2002;122(4):1400-1406.

38. Dunleavy M, Bradford A, O'Halloran KD. Oxidative stress impairs upper airway muscle endurance in an animal model of sleep-disordered breathing. Adv Exp Med Biol. 2008;605:458-462.

39. Pae EK, Wu J, Nguyen D, Monti R, Harper RM. Geniohyoid muscle properties and myosin heavy chain composition are altered after short-term intermittent hypoxic exposure. J Appl Physiol (1985). 2005;98(3):889-894.

40. Liu YH, Huang Y, Shao X. Effects of estrogen on genioglossal muscle contractile properties and fiber-type distribution in chronic intermittent hypoxia rats. Eur J Oral Sci. 2009;117(6):685-690.

41. Jia SS, Liu YH. Down-regulation of hypoxia inducible factor-1alpha: a possible explanation for the protective effects of estrogen on genioglossus fatigue resistance. Eur J Oral Sci. 2010;118(2):139-144.

42. Ding W, Liu Y. Genistein attenuates genioglossus muscle fatigue under chronic intermittent hypoxia by down-regulation of oxidative stress level and up-regulation of antioxidant enzyme activity through ERK1/2 signaling pathway. Oral Dis. 2011;17(7):677-684.

43. Wang WJ, Lu G, Ding N, Huang HP, Ding WX, Zhang XL. Adiponectin alleviates contractile dysfunction of genioglossus in rats exposed to chronic intermittent hypoxia. Chin Med J (Engl). 2013;126(17):3259-3263.

44. Zhou J, Liu Y. Effects of genistein and estrogen on the genioglossus in rats exposed to chronic intermittent hypoxia may be HIF-1alpha dependent. Oral Dis. 2013;19(7):702-711.

45. Huang H, Jiang X, Dong Y, et al. Adiponectin alleviates genioglossal mitochondrial dysfunction in rats exposed to intermittent hypoxia PLoS One. 2014;9(10):e109284.

46. Skelly JR, Edge D, Shortt CM, Jones JF, Bradford A, O'Halloran KD. Tempol ameliorates pharyngeal dilator muscle dysfunction in a rodent model of chronic intermittent hypoxia. Am J Respir Cell Mol Biol. 2012;46(2):139-148.

47. Skelly JR, Edge D, Shortt CM, Jones JF, Bradford A, O'Halloran KD. Respiratory control and sternohyoid muscle structure and function in aged male rats: decreased susceptibility to chronic intermittent hypoxia. Respir Physiol Neurobiol. 2012;180(2-3):175-182.

48. Series F, Cote C, Simoneau JA, St Pierre S, Marc I. Upper airway collapsibility, and contractile and metabolic characteristics of musculus uvulae. FASEB J. 1996;10(8):897-904.

49. Petrof BJ, Pack AI, Kelly AM, Eby J, Hendricks JC. Pharyngeal myopathy of loaded upper airway in dogs with sleep apnea. $J$ Appl Physiol (1985). 1994;76(4):1746-1752.

50. Shortt CM, Fredsted A, Chow HB, et al. Reactive oxygen species mediated diaphragm fatigue in a rat model of chronic intermittent hypoxia. Exp Physiol. 2014;99(4):688-700.

51. Clanton TL, Wright VP, Reiser PJ, Klawitter PF, Prabhakar NR. Selected contribution: improved anoxic tolerance in rat diaphragm following intermittent hypoxia. J Appl Physiol (1985). 2001;90(6):2508-2513.

52. Giordano C, Lemaire C, Li T, Kimoff RJ, Petrof BJ. Autophagy-associated atrophy and metabolic remodeling of the mouse diaphragm after short-term intermittent hypoxia. PLoS One. 2015;10(6):e0131068.
53. Shortt CM, Fredsted A, Bradford A, O'Halloran KD. Diaphragm muscle remodeling in a rat model of chronic intermittent hypoxia. J Histochem Cytochem. 2013;61(7):487-499.

54. McGuire M, MacDermott M, Bradford A. Effects of chronic intermittent asphyxia on rat diaphragm and limb muscle contractility. Chest. 2003;123(3):875-881.

55. Farkas GA, McCormick KM, Gosselin LE. Episodic hypoxia exacerbates respiratory muscle dysfunction in $\mathrm{DMD}(\mathrm{mdx})$ mice. Muscle Nerve. 2007;36(5):708-710.

56. Sawnani H, Thampratankul L, Szczesniak RD, Fenchel MC, Simakajornboon N. Sleep disordered breathing in young boys with Duchenne muscular dystrophy. J Pediatr. 2015;166(3):640-645. e641.

57. Ray AD, Magalang UJ, Michlin CP, et al. Intermittent hypoxia reduces upper airway stability in lean but not obese Zucker rats. Am J Physiol Regul Integr Comp Physiol. 2007;293(1):R372-R378.

58. McDonald FB, Edge D, O'Halloran KD. Chronic nitric oxide synthase inhibition does not impair upper airway muscle adaptation to chronic intermittent hypoxia in the rat. Prog Brain Res. 2014;212:237-251.

59. Huang Y, Liu YH. Effects of phytoestrogens on genioglossus contractile properties in ovariectomized rats exposed to chronic intermittent hypoxia may be independent of their estrogenicity. Eur J Oral Sci. 2011;119(2):128-135.

60. Lu Y, Liu Y, Li Y. Comparison of natural estrogens and synthetic derivative on genioglossus function and estrogen receptors expression in rats with chronic intermittent hypoxia. J Steroid Biochem Mol Biol. 2014;140:71-79.

61. Li W, Liu YH. Effects of phytoestrogen genistein on genioglossus function and oestrogen receptors expression in ovariectomized rats. Arch Oral Biol. 2009;54(11):1029-1034.

62. Hunter SK. The relevance of sex differences in performance fatigability. Med Sci Sports Exerc. 2016;48(11):2247-2256.

63. Hunter SK. Sex differences in fatigability of dynamic contractions. Exp Physiol. 2016;101(2):250-255.

64. Yoon T, Doyel R, WiduleC, Hunter SK. Sex differences with aging in the fatigability of dynamic contractions. Exp Gerontol. 2015;70:1-10.

65. Redline S, Kump K, Tishler PV, Browner I, Ferrette V. Gender differences in sleep disordered breathing in a community-based sample. $\mathrm{Am}$ J Respir Crit Care Med. 1994;149(3 Pt 1):722-726.

66. Young T, Finn L, Austin D, Peterson A. Menopausal status and sleepdisordered breathing in the Wisconsin Sleep Cohort Study. Am J Respir Crit Care Med. 2003;167(9):1181-1185.

67. Young T, Palta M, Dempsey J, Skatrud J, Weber S, Badr S. The occurrence of sleep-disordered breathing among middle-aged adults. $N$ Engl J Med. 1993;328(17):1230-1235.

68. Senaratna CV, Perret JL, Lodge CJ, et al. Prevalence of obstructive sleep apnea in the general population: a systematic review. Sleep Med Rev. Epub 2016 Jul 18

69. Kimoff RJ. Upperairway myopathy is important in the pathophysiology of obstructive sleep apnea. J Clin Sleep Med. 2007;3(6):567-569.

70. Griggs GA, Findley LJ, Suratt PM, Esau SA, Wilhoit SC, Rochester DF. Prolonged relaxation rate of inspiratory muscles in patients with sleep apnea. Am Rev Respir Dis. 1989;140(3):706-710.

71. Chien MY, Wu YT, Lee PL, Chang YJ, Yang PC. Inspiratory muscle dysfunction in patients with severe obstructive sleep apnoea. Eur Respir J. 2010;35(2):373-380.

72. El-Khoury R, O'Halloran KD, Bradford A. Effects of chronic hypobaric hypoxia on contractile properties of rat sternohyoid and diaphragm muscles. Clin Exp Pharmacol Physiol. 2003;30(8):551-554.

73. Gamboa JL, Andrade FH. Muscle endurance and mitochondrial function after chronic normobaric hypoxia: contrast of respiratory and limb muscles. Pflugers Arch. 2012;463(2):327-338.

74. Degens H, Bosutti A, Gilliver SF, Slevin M, van Heijst A, Wust RC. Changes in contractile properties of skinned single rat soleus and diaphragm fibres after chronic hypoxia. Pflugers Arch. 2010;460(5):863-873.

75. Lewis P, Sheehan D, Soares R, Coelho AV, O'Halloran KD. Redox remodeling is pivotal in murine diaphragm muscle adaptation to chronic sustained hypoxia. Am J Respir Cell Mol Biol. 2016;55(1):12-23. 
76. Lewis P, McMorrow C, Bradford A, O'Halloran KD. Improved tolerance of acute severe hypoxic stress in chronic hypoxic diaphragm is nitric oxide-dependent. J Physiol Sci. 2015;65(5):427-433.

77. Lewis P, Sheehan D, Soares R, Varela Coelho A, O'Halloran KD. Chronic sustained hypoxia-induced redox remodeling causes contractile dysfunction in mouse sternohyoid muscle. Front Physiol. 2015;6:122.

78. Burns DP, O'Halloran KD. Evidence of hypoxic tolerance in weak upper airway muscle from young mdx mice. Respir Physiol Neurobiol. 2016;226:68-75.

79. O'Leary AJ, O'Halloran KD. Diaphragm muscle weakness and increased UCP-3 gene expression following acute hypoxic stress in the mouse. Respir Physiol Neurobiol. 2016;226:76-80.

80. Mortola JP, Naso L. Electrophoretic analysis of contractile proteins of the diaphragm in chronically hypoxic rats. Am J Physiol. 1995;269(3 Pt 1):L371-L376.

81. Lewis $\mathrm{P}, \mathrm{O}$ 'Halloran KD. Sex differences in murine sternohyoid muscle tolerance of acute severe hypoxic stress. Physiol Res. 2016;65(5):843-851.

82. Williams R, Lemaire P, Lewis $\mathrm{P}$, et al. Chronic intermittent hypoxia increases rat sternohyoid muscle NADPH oxidase expression with attendant modest oxidative stress. Front Physiol. 2015;6:15.

83. Skelly JR, Bradford A, Jones JF, O’Halloran KD. Superoxide scavengers improve rat pharyngeal dilator muscle performance. Am J Respir Cell Mol Biol. 2010;42(6):725-731.

84. Shortt CM, O'Halloran KD. Hydrogen peroxide alters sternohyoid muscle function. Oral Dis. 2014;20(2):162-170.

85. Zhang XF, Huang HP, Ding WX, et al. Adiponectin protects the genioglossus of rats against chronic intermittent hypoxia-induced injury via inhibition of endoplasmic reticulum stress. Chin Med J (Engl). 2013;126(17):3270-3275.

86. Zhang XF, Wang YH, Li Q, et al. Changes in genioglossus and their association with serum adiponectin levels in rats subjected to chronic intermittent hypoxia. Chin Med J (Engl). 2010;123(16):2249-2253.

87. Huang H, Zhang X, Ding N, Li Q, Min Y, Zhang X. Effects of chronic intermittent hypoxia on genioglossus in rats. Sleep Breath. 2012;16(2):505-510

88. Dominguez-Alvarez M, Gea J, Barreiro E. Inflammatory events and oxidant production in the diaphragm, gastrocnemius, and blood of rats exposed to chronic intermittent hypoxia: therapeutic strategies. J Cell Physiol. 2016;232(5):1165-1175.

89. Gamboa JL, Andrade FH. Mitochondrial content and distribution changes specific to mouse diaphragm after chronic normobaric hypoxia. Am J Physiol Regul Integr Comp Physiol. 2010;298(3):R575-R583.

90. Borras C, Sastre J, Garcia-Sala D, Lloret A, Pallardo FV, Vina J. Mitochondria from females exhibit higher antioxidant gene expression and lower oxidative damage than males. Free Radic Biol Med. 2003;34(5):546-552.

91. Derbre F, Ferrando B, Gomez-Cabrera MC, et al. Inhibition of xanthine oxidase by allopurinol prevents skeletal muscle atrophy: role of p38 MAPKinase and E3 ubiquitin ligases. PLoS One. 2012;7(10):e46668.

92. Murciano D, Aubier M, Lecocguic Y, Pariente R. Effects of theophylline on diaphragmatic strength and fatigue in patients with chronic obstructive pulmonary disease. N Engl J Med. 1984;311(6):349-353.

93. Decramer M. Hyperinflation and respiratory muscle interaction. Eur Respir J. 1997;10(4):934-941.
94. Similowski T, Yan S, Gauthier AP, Macklem PT, Bellemare F. Contractile properties of the human diaphragm during chronic hyperinflation. N Engl J Med. 1991;325(13):917-923.

95. Haddad GG, Akabas SR. Adaptation of respiratory muscles to acute and chronic stress. Considerations on energy and fuels. Clin Chest Med. 1986;7(1):79-89.

96. Guenette JA, Romer LM, Querido JS, et al. Sex differences in exerciseinduced diaphragmatic fatigue in endurance-trained athletes. $J \mathrm{Appl}$ Physiol (1985). 2010;109(1):35-46.

97. Roussos CS, Macklem PT. Diaphragmatic fatigue in man. J Appl Physiol Respir Environ Exerc Physiol. 1977;43(2):189-197.

98. Babcock MA, Johnson BD, Pegelow DF, Suman OE, Griffin D, Dempsey JA. Hypoxic effects on exercise-induced diaphragmatic fatigue in normal healthy humans. J Appl Physiol (1985). 1995;78(1):82-92.

99. Vogiatzis I, Georgiadou O, Koskolou M, Athanasopoulos D, et al. Effects of hypoxia on diaphragmatic fatigue in highly trained athletes. J Physiol. 2007;581(Pt 1):299-308.

100. Verges S, Bachasson D, Wuyam B. Effect of acute hypoxia on respiratory muscle fatigue in healthy humans. Respir Res. 2010;11:109.

101. Jardim J, Farkas G, Prefaut C, Thomas D, Macklem PT, Roussos C. The failing inspiratory muscles under normoxic and hypoxic conditions. Am Rev Respir Dis. 1981;124(3):274-279.

102. Ward ME, Eidelman D, Stubbing DG, Bellemare F, Macklem PT. Respiratory sensation and pattern of respiratory muscle activation during diaphragm fatigue. J Appl Physiol (1985). 1988;65(5):2181-2189.

103. Reid MB. Reactive oxygen species as agents of fatigue. Med Sci Sports Exerc. 2016;48(11):2239-2246.

104. Ferreira LF, Reid MB. Muscle-derived ROS and thiol regulation in muscle fatigue. J Appl Physiol (1985). 2008;104(3):853-860.

105. Morgan BJ, Adrian R, Wang ZY, Bates ML, Dopp JM. Chronic intermittent hypoxia alters ventilatory and metabolic responses to acute hypoxia in rats. J Appl Physiol (1985). 2016;120(10):1186-1195.

106. Sollanek KJ, Burniston JG, Kavazis AN, et al. Global proteome changes in the rat diaphragm induced by endurance exercise training. PLoS One. 2017;12(1):e0171007.

107. Powers SK, Shanely RA. Exercise-induced changes in diaphragmatic bioenergetic and antioxidant capacity. Exerc Sport Sci Rev. 2002;30(2):69-74

108. Vrabas IS, Dodd SL, Powers SK, et al. Endurance training reduces the rate of diaphragm fatigue in vitro. Med Sci Sports Exerc. 1999; 31(11):1605-1612.

109. Vincent HK, Shanely RA, Stewart DJ, et al. Adaptation of upper airway muscles to chronic endurance exercise. Am J Respir Crit Care Med. 2002;166(3):287-293.

110. Garcia-Cazarin ML, Gamboa JL, Andrade FH. Rat diaphragm mitochondria have lower intrinsic respiratory rates than mitochondria in limb muscles. Am J Physiol Regul Integr Comp Physiol. 2011;300(6):R1311-R1315.

111. El-Khoury R, Bradford A, O’Halloran KD. Chronic hypobaric hypoxia increases isolated rat fast-twitch and slow-twitch limb muscle force and fatigue. Physiol Res. 2012;61(2):195-201.

112. Selsby JT, Acosta P, Sleeper MM, Barton ER, Sweeney HL. Long-term wheel running compromises diaphragm function but improves cardiac and plantarflexor function in the mdx mouse. J Appl Physiol (1985). 2013;115(5):660-666
Hypoxia

\section{Publish your work in this journal}

Hypoxia is an international, peer-reviewed, open access journal that aims to improve understanding of the biological response to hypoxia. The journal will publish original research articles, reviews, methodological advances, clinical studies, and expert opinions that identify developments in the regulation of the physiological and pathological responses to Submit your manuscript here: https://www.dovepress.com/hypoxia-journal

\section{Dovepress}

hypoxia and in the therapeutic targeting of hypoxia-responsive pathways. The manuscript management system is completely online and includes a very quick and fair peer-review system, which is all easy to use. Visit http://www.dovepress.com/testimonials.php to read real quotes from published authors. 